\title{
Vulnerabilidad de humedales altoandinos ante procesos de cambio: tendencias del análisis*
}

\author{
Mónica Patricia Valencia Rojas* \\ Apolinar Figueroa Casas ${ }^{* * *}$
}

Recibido: 19/11/2013 - Aceptado: 12/12/2014

\section{RESUMEN}

Los humedales de alta montaña son ecosistemas vulnerables que juegan un papel fundamental en la dinámica hidrológica, cultural, ecológica y socioeconómica de la región andina colombiana, que siguen deteriorándose por causas asociadas al cambio climático y a la presión generada por actividades antrópicas. Su conservación ha despertado el interés de múltiples actores que buscan elementos conceptuales, herramientas de evaluación y alternativas de gestión que permitan direccionar de mejor manera los procesos de manejo de estos ecosistemas a través de nuevas visiones y comprensiones en torno a la mitigación y adaptación a los direccionadores de cambio. En el presente documento se busca hacer una revisión de algunos referentes en torno a los procesos de degradación de los humedales altoandinos, exponer las tendencias de los análisis de la vulnerabilidad y resaltar el reto actual de desarrollar los análisis a través de una visión que integre lo social y lo biofísico, a partir de un enfoque sistémico de tan importantes sistemas naturales, con la finalidad de introducir la visión de los sistemas socio-ecológicos o socio-ecosistemas como una alternativa ya conocida, pero que debe ser desarrollada y apropiada para su implantación y comprensión por las comunidades humanas.

Palabras clave: interdisciplinariedad, laguna, páramo, socio-ecosistemas.

\footnotetext{
Esta revisión es producto de la investigación doctoral titulada "Vulnerabilidad de las lagunas de páramo a procesos degradativos de origen antrópico incorporando la complejidad ambiental del territorio (departamento del Cauca, Colombia)", financiada por Colciencias a través del Programa de Becas Nacionales [1]

**Ambientales Universidad del Cauca. Email: mvalenciarojas@unicauca.edu.co

**** Doctor en Ciencias (PhD). Director Grupo GEA, Profesor titular Programa de Biología Universidad del Cauca. Email: apolinarfigueroa@unicauca.edu.co
} 


\title{
Vulnerability of andean wetlands to change processes: trends analysis
}

\begin{abstract}
The Andean wetlands are fragile ecosystems that play a key role in hydrological, cultural, ecological and socioeconomic dynamics of the Colombian Andean region; to continue to deteriorate for reasons associated with climate change and the pressure generated by human activities. Its preservation has aroused the interest of many actors who seek conceptual, assessment tools and management options that allow the management processes of these ecosystems through new insights and understandings about mitigation and adaptation to future changes. This paper seeks a review of some references about the degradation processes of the Andean wetlands, exhibit trends in vulnerability analysis and highlight the current challenge of developing the analysis through a vision that integrates social and biophysical, from a systemic approach of important natural systems. In order to introduce the vision of socio-ecological systems or socio-ecosystems as a known alternative, but that must be developed and appropriate for implementation and understanding of human communities.
\end{abstract}

Keywords: interdisciplinarity, lagoon, paramo, socio-ecosystems. 


\section{INTRODUCCIÓN}

Los humedales están estrechamente relacionados con el bienestar humano, debido a su trascedente aporte en más del $40 \%$ en los servicios ecosistémicos del planeta por año, incluyendo protección contra las inundaciones, mejora en la calidad del agua, almacenamiento de carbono, el ciclo de nutrientes, entre otros $[1,2,3]$. Asimismo, cumplen funciones vitales tales como el almacenamiento de agua, la conservación de la biodiversidad, y el control de la erosión, y constituyen la base de beneficios económicos para la pesca y la agricultura, además de ser un elemento importante del patrimonio cultural de diversos territorios $[3,4]$.

Gracias a la provisión constante de agua, dadas las condiciones ecológicas y climatológicas de los ecosistemas de alta montaña, la Región Andina tiene una constante oferta de agua especialmente para el soporte ecológico de estos sistemas naturales y la oferta ambiental. Estas condiciones están sometidas a una demanda hídrica constante como lo muestran los registros nacionales, los cuales indican que alcanzó para el 2008 un orden de $35.877 \mathrm{Mm}^{3}$, donde el sector agrícola empleó cerca de la mitad de la demanda hídrica (54,03\%); una cuarta parte se empleó en el sector energético $(19,44 \%)$; y la otra cuarta parte se divide entre los sectores pecuario y acuícola $(13,4$ $\%)$, doméstico (7,26 \%) industrial (4,40\%) y de servicios (1,47\%) [5].

Frente a escenarios climáticos presentados en la segunda comunicación nacional de Cambio Climático de Colombia [6] se espera: un aumento de la temperatura media entre 2 y $4{ }^{\circ} \mathrm{C}$ al año 2070; una modificación de las condiciones hidrológicas con reducción de las precipitaciones en algunas regiones de hasta un $30 \%$; un aumento en la recurrencia de eventos extremos (Niño y Niña), y una creciente incidencia de emergencias asociadas al clima, ya sean estas por efectos de aumento o por disminución en la oferta, por afectación de los ritmos de escorrentía superficial y humedad del suelo, y por amenaza de eventos, como las inundaciones y sequías [7, 8], situaciones que son inquietantes y nos enfrentan a diseñar medidas de gestión y de ordenamiento ambiental del territorio que, aunque difíciles, deben ser abordadas de manera urgente.

Se espera que el cambio climático provoque una intensificación de las temperaturas y una afectación en el ciclo hidrológico global, con alteraciones hidrológicas regionales y cambios fundamentales en los humedales especialmente en sus condiciones hidrológicas (profundidad e hidroperíodo), lo que hace más extremo el estrés térmico y propicia cambios en el uso del suelo; incluso es posible que el cambio climático se traduzca en variaciones en las funciones y distribución regional de los humedales interiores [3]. Pero una alteración drástica en el ciclo hidrológico, asociada a la pérdida de glaciares, variación en la precipitación, temperatura y cambio de coberturas vegetales, significaría que los humedales de alta montaña modifiquen su papel como eslabón en 
la dinámica del agua para la Región Andina, alterando sus funciones ecosistémicas y servicios asociados al suministro, la regulación, su valor cultural y de soporte que brindan los humedales.

Actualmente, muchas de las evaluaciones del estado de estos ecosistemas desde el enfoque sistémico han involucrado elementos propios de la ecología funcional, como son la vulnerabilidad, la resiliencia, la capacidad de adaptación y la transformabilidad como aspectos conceptuales y prácticos indispensables para entender a fondo su dinámica, para poder valorar de forma más integral los impactos a que están sometidos y discernir las diferentes respuestas a procesos de presión y alteración que generalmente están sobrepasando su capacidad de carga. Estudios de las propiedades emergentes del ecosistema (diversidad, heterogeneidad, resiliencia, vulnerabilidad) se están desarrollando de diversas formas, con trasfondos conceptuales y metodológicos diferentes, que limitan las comparaciones y la formulación de propuestas de gestión ambiental basadas en la sostenibilidad del territorio. En este sentido, a continuación se presenta la revisión de los procesos de afectación de los humedales de alta montaña, las tendencias en el estudio de la vulnerabilidad hasta llegar a la visión de los sistemas socio-ecológicos como una alternativa para el estudio de las propiedades emergentes de los humedales.

\section{PROCESOS DE AFECTACIÓN EN LOS HUMEDALES DE ALTA MONTAÑA}

Los procesos de afectación humana en los humedales no son independientes de la dinámica natural de estos sistemas [9]. En la actualidad, los humedales están sufriendo un acelerado deterioro, evidente no solo en la pérdida de área sino también en la disminución de atributos por la afectación directa o indirecta de sus procesos fundamentales; entre estos se tienen los cambios descritos por Mitsch y Gosselink (2000) destacándose las modificaciones hidro-geomorfológicas a través del clima, las condiciones del terreno, y las actividades antrópicas (cf. drenajes, infraestructuras); donde la hidrología del humedal cambia las propiedades químicas y físicas del sistema natural (cf. pH, oxígeno, nutrientes, contaminantes); esta condición altera significativamente su biota. Lo descrito impacta su estructura y función, y se comporta como un ciclo con realimentación. Estos tipos de procesos generan estrés y llevan al humedal a su degradación, lo que está asociado a la magnitud y dirección de la antropización y, por ende, al impacto generado en relación con su entorno.

El deterioro de los humedales colombianos se ha exacerbado debido a la intervención dada en los páramos y los piedemontes cordilleranos andinos, ocupación y uso que comenzaron en el siglo antepasado, y desde entonces, la influencia y la "antropización" de estos ha venido en aumento [10]. En numerosas regiones paramunas colombianas 
se adelantan labores productivas inapropiadas respecto a la verdadera vocación de uso del suelo dictada por sus características físicas y bióticas, a través de concesiones mineras (oro, carbón), monocultivos forestales (pino, eucalipto), agricultura intensiva, introducción de especies, ganadería, extracción intensiva de leña y materiales (bambúes, paja, juncos), turismo masivo, entre otras [11-14].

Los cambios de los humedales están directa o indirectamente asociados a patrones de distribución de los asentamientos humanos. La transformación de una economía basada en la agricultura extensiva y el pastoreo, hacia un sistema dominado por el monocultivo y la concentración poblacional en las últimas décadas es responsable de la alteración y degradación de importantes sistemas acuáticos como, por ejemplo, los del altiplano Cundiboyacense [15, 16], la laguna de Sonso y los sistemas de humedales riparianos en el Valle del Cauca [17]; de sistemas altoandinos como la laguna de Fúquene en Cundinamarca [18], lago de la Tota en Boyacá [19] y las lagunas de San Rafael [20, 21] y de Calvache [20] en el departamento del Cauca, entre otros sistemas alterados.

Dicho deterioro se ha hecho notorio en los eventos de alta pluviosidad experimentados en el país cada año, en donde la ausencia/deficiencia en las funciones de las lagunas paramunas, como sistemas buffer y reguladores en el ciclo hidrológico en la alta montaña, han disminuido un eslabón trascendental en la dinámica hidrológica de la región andina. Con ello se ha causado el aumento en la cantidad de agua que desciende de las partes altas, incremento en la lixiviación y la escorrentía, los deslizamientos del suelo, entre otros fenómenos. Tal situación ha generado la multiplicación de inundaciones y sequías, haciendo evidente las interrelaciones existentes entre el talado de los bosques en las partes altas de las cuencas hidrográficas, las transformaciones de las lagunas paramunas y los desastres naturales que han tenido que afrontar algunas comunidades humanas por estos motivos.

Ello trae, como consecuencia, drásticas inundaciones que originan desplazamiento humano, daños en infraestructuras y tierras productivas, afectación de la seguridad alimentaria y, por lo tanto, impacto negativo en la capacidad competitiva de la Región Andina y el país en general. En los últimos tres años, Colombia ha experimentado fuertes inundaciones asociadas a la transformación de la capacidad de retención y descarga de lagunas altoandinas como la Cocha y Fúquene, situación agravada por la debilidad en el conocimiento de las dinámicas ecológicas (cf. pulsos de inundación) en las llanuras inundables, una planificación territorial inadecuada en zonas medias/ bajas de las cuencas y la falta de prevención ante efectos drásticos de los fenómenos climáticos (el Niño y la Niña). Las alteraciones de los sistemas lénticos y lóticos tienen, como resultado, importantes desastres socio-naturales, como los registrados en los planos inundables del río Bogotá y el departamento el Atlántico (cf. canal del Dique), 
entre otros lugares impactados por inundaciones de grandes ríos andinos como lo son el Cauca y el Magdalena [22].

\section{TENDENCIA DE LOS ANÁLISIS DE VULNERABILIDAD DE LOS HUMEDALES ANTE PRO- CESOS DE CAMBIO}

Los humedales son reconocidos por su vulnerabilidad a los eventos naturales [23, $24]$ y a las continuas presiones humanas $[3,25]$. Cuando se habla de vulnerabilidad, se está expresando de manera general la susceptibilidad al daño, y se caracteriza a menudo en términos de uno o más de los siguientes elementos: la sensibilidad y/o la exposición de un sistema (personas o lugar) a las crisis, tensiones o perturbaciones, el estado del sistema en relación con un umbral de daño, y la capacidad del sistema para adaptarse a condiciones cambiantes [23, 26-31]; pero la definición varía de acuerdo con la disciplina que la estudia y el área de aplicación [32]. La vulnerabilidad se aborda en este documento, como el grado en el cual un sistema es probable que sufra daños debido a la exposición a una amenaza especifica o estrés [26,33].

La conceptualización de la vulnerabilidad ha transitado a través de tres décadas desde una perspectiva monodisciplinar (desde la geografía y las ciencias sociales), hasta una visión multidisplinar al trabajar a través de la integración de diversas áreas del conocimiento (fusión de ideas en amenazas naturales, marcos políticos-económicos y ecología política), y llega al abordaje interdisciplinario el cual involucra la interacción de las diferentes partes del sistema concebido (sistemas socio-ecológicos), hasta arribar, en los últimos años, al desarrollo de su conceptualización y procedimientos metodológicos a través de un abordaje transdisciplinar que involucra la generación de nuevos elementos.

No obstante, en la literatura predominan tres perspectivas clásicas para el estudio de la vulnerabilidad (biofísica, social, integrada); tal y como lo plantea Soares [34], estas tres formas tienen elementos diferenciales tales como el punto focal del análisis, los tipos de pregunta a responder, los sistemas de análisis abordados y los periodos de tiempo, aspectos que delimitan el concepto a emplear, el modelo conceptual y los métodos para valorarla según el estudio.

Tanto su definición como los enfoques empleados para su abordaje continúan creciendo, especialmente bajo la mirada integradora de los elementos humanos y naturales que componen un sistema, acompañados de diversos factores internos y externos que delimitan su interacción y dinámica [35].

La valoración de la vulnerabilidad y del riesgo varía de acuerdo con el marco conceptual empleado en el análisis. Existen diferentes marcos para esto, por ejemplo i) medios de vida sostenibles [36], ii) modelo de presión liberación (PAR) [37], 
iii) la doble estructura de la vulnerabilidad [38], iv) modelo vulnerabilidad de lugar [39], v) modelo de riesgo amenaza (HR), y vi) vulnerabilidad desde el cambio global [26], entre otros. La mayoría de los modelos conceptuales está orientada a conocer la vulnerabilidad social y en algunos casos incluye la vulnerabilidad biofísica (tabla 1). Esto obedece a que están fundamentados en el estudio del riesgo ante una amenaza específica (especialmente de origen natural) y abordados tradicionalmente desde la ingeniería, la geografía y las ciencias sociales, a diferencia del modelo de cambio global que está fundamentado en los principios de la resiliencia de los sistemas, que están enfrentando diferentes cambios.

Tabla 1. Comparación de los modelos de vulnerabilidad tradicionales

\begin{tabular}{|c|c|c|c|c|c|c|c|}
\hline & & $\begin{array}{c}\text { Medios } \\
\text { de vida } \\
\text { Sostenibles } \\
\text { Chambers } \\
\text { \& Conway } \\
\text { (1992) } \\
\end{array}$ & $\begin{array}{c}\text { Presión } \\
\text {-liberación } \\
\text { Blaikie et al } \\
\text { (1994). }\end{array}$ & $\begin{array}{c}\text { Doble } \\
\text { estructura } \\
\text { Bohle et al., } \\
\text { (1994) }\end{array}$ & $\begin{array}{c}\text { De Lugar } \\
\text { Cutter } \\
(1996)\end{array}$ & $\begin{array}{c}\text { Riesgo } \\
\text {-Amenaza } \\
\text { Davidson } \\
\text { (1997) }\end{array}$ & $\begin{array}{c}\text { Desde } \\
\text { Cambio } \\
\text { Global } \\
\text { Turner } \\
(2003)\end{array}$ \\
\hline & Vulnerabilidad Social & $\mathrm{X}$ & $\mathrm{X}$ & $\mathrm{X}$ & $\mathrm{X}$ & $\mathrm{X}$ & \\
\hline & Vulnerabilidad Biofísica & & $\mathrm{X}$ & & $\mathrm{X}$ & $\mathrm{X}$ & \\
\hline $\begin{array}{l}\text { Alcan- } \\
\text { ce del } \\
\text { análisis }\end{array}$ & $\begin{array}{l}\text { Vulnerabilidad Integrada } \\
\text { (Social y Biofísica) }\end{array}$ & & & & & & $\mathrm{X}$ \\
\hline & Amenaza & & $\mathrm{X}$ & & $\mathrm{X}$ & $\mathrm{X}$ & \\
\hline & Riesgo & $\mathrm{X}$ & $\mathrm{X}$ & & $X$ & $\mathrm{X}$ & \\
\hline & Multiescalar & & & & $X$ & $\mathrm{X}$ & $\mathrm{X}$ \\
\hline deracio- & Multidimensional & & $\mathrm{X}$ & & $\mathrm{X}$ & $\mathrm{X}$ & \\
\hline nes & Multitensor & & & & & $\mathrm{X}$ & $\mathrm{X}$ \\
\hline
\end{tabular}

Fuente: [1]

En términos de escala, todos implican el individuo, y la familia (elementos micro); pero tan solo el modelo de lugar y el de cambio global son mutiescalares al trabajar elementos micro y macro (territorio, ecosistema) en su estimación. En cuanto a las dimensiones, todos los modelos incluyen múltiples dimensiones de la vulnerabilidad; esto significa que incluyen, por lo menos, tres de las diversas dimensiones de la vulnerabilidad, que son comúnmente identificadas a través de la literatura (por ejemplo, social, económica, ambiental y política); sin embargo, no son vistas de manera sistémica al considerar los diferentes elementos constituyentes del sistema y la interacción entre ellos. Estos modelos no trabajan diferentes tensores a la vez, es decir, no son multitensores porque no incluyen la influencia de diversos factores naturales y antrópicos sobre el sistema. A pesar de que el modelo de cambio global es el único de estos referentes 
que tiene una visión más integradora, carece de ser multitensor, no hay claridad en las dimensiones a tener en cuenta y la resiliencia es uno de los elementos que constituyen la vulnerabilidad. Además, ninguno de los modelos expuesto involucra los aspectos culturales de las comunidades humanas.

Atendiendo lo expuesto anteriormente, se evidencia que los modelos empleados comúnmente para el análisis de la vulnerabilidad no permiten acercarse a la interacción ser humano-naturaleza de una manera sistémica, e incluir diversos promotores de cambio (stressors) a través de diferentes escalas espaciales y temporales para dar cabida a las interdependencias que originan la dinámica de vulnerabilidad. Es frecuente encontrar en los análisis de cambio climático en humedales, los modelos de presión liberación, riesgo y vulnerabilidad desde el cambio global, como es el caso de los estudios de Franco et al., 2011 y Gitay et al., 2001 [40-42].

Si bien cada uno de los modelos conceptuales mencionados tienen características propias y cuentan con sus ventajas y desventajas [26, 43] de acuerdo con el área de aplicación, existe un material bibliográfico suficiente que permite elegir o seleccionar el campo de acción; no hay una receta estándar para medir la vulnerabilidad de los sistemas, ni una mejor forma de conceptualizarla o trabajarla [44]. Pero con la intención de abordar el reto que significa trabajar la vulnerabilidad desde una visión sistémica, el desafío debe ser abordado desde una perspectiva interdisciplinaria, mucho más allá de las visiones disciplinares y multidisciplinares trabajadas en los últimos años, con el propósito de construir una mirada integradora, que reconozca al hombre como el principal factor transformador.

Esta visión es la clave para entender la complejidad de la problemática ambiental asociada a los humedales altoandinos, y en especial a las lagunas de páramo, complejidad que nace de la articulación entre la naturaleza, la técnica y la cultura, como lo plantea Leff [45], como un proceso de comunicación de saberes, de intercambio de experiencias y complementación de conocimientos que apunte hacia la producción de otras formas de comprensión de una problemática ambiental.

En este sentido, la vulnerabilidad abordada desde la complejidad ambiental debe incorporar elementos procedentes de diversos componentes del sistema (los más importantes/de mejor indicación), como lo son los aspectos sociales (cf. cosmovisión, patrones culturales, saberes), aspectos económicos (cf. productividad, ingresos); legislación (cf. política humedales interiores, cabildo indígena); dinámica natural (cf. sucesión natural, variabilidad climática); dinámica social (cf. actividades antrópicas) y ecofisiología (cf. características propias del humedal), entre otros. 


\section{VULNERABILIDAD DE LAS LAGUNAS DESDE LOS SISTEMAS SOCIO-ECOLÓGICOS}

Para abordar la vulnerabilidad de las lagunas de páramo a través de una mirada sistémica es necesario identificar sus dinámicas, teniendo en cuenta los diferentes factores explicativos (cf. natural, cultural, económico) e integrando diversas disciplinas de las ciencias naturales/formales como la biología, la física y las matemáticas; las ciencias nomotéticas como la antropología, la sociología, y la economía; y los saberes como las cosmovisiones, las lógicas de vida, los conocimientos empíricos, entre otros, para su interpretación y comprensión.

Especialmente, ante los diversos impactos ambientales producto del ser humano y su consecuente alteración de los sistemas naturales, se ha reconocido la necesidad de pensar más allá de una o varias disciplinas unidas, para evolucionar a marcos conceptuales integrales y transdiciplinarios. Dichos marcos reconocen que las sociedades y la naturaleza son interdependientes y deben ser abordados de una manera integrada, para lo cual se propone emplear el modelo de los Sistemas Socio-Ecológicos (SSE) $[26,46,47]$.

Este modelo considera las relaciones dinámicas entre las condiciones biofísicas, sociales, culturales y políticas (entre otros factores) a través de escalas temporales y espaciales específicas; todo con el fin de explicar sus interrelaciones ambientales complejas, en función de su exposición, sensibilidad y capacidad adaptativa. Téngase presente que bajo este enfoque se reconocen, además, la resiliencia, la capacidad adaptativa (capacidad del sistema a aprender combinar experiencias y conocimiento) y la transformabilidad (capacidad de crear un nuevo sistema totalmente nuevo cuando el actual sea insostenible) como características propias de un sistema, los cuales son determinantes en su capacidad para adaptarse o beneficiarse de los cambios [48]. Estas características son complementarias y no existe realmente un comportamiento inverso entre la resiliencia y la vulnerabilidad [48-50]; si no por el contrario, comparten elementos que propician su capacidad de respuesta y de auto-organización del mismo.

Desde la perspectiva de las ciencias ambientales, los sistemas socio-ecológicos asociados a las lagunas de páramo se comportan como sistemas complejos, al concebirlos como un todo organizado, un sistema dinámico que está compuesto, a su vez, de subsistemas los cuales transitan a través de diferentes estados de equilibrio, atribuidos a sus continuas perturbaciones y sus procesos de adaptación y reorganización. En este orden de ideas, el enfoque de los sistemas socio-ecológicos, permite trabajar la problemática ambiental que presentan las lagunas de páramo ante los procesos antropogénicos, por medio de un análisis que integre su carácter dinámico, la multiplicidad de relaciones de sus elementos 
constitutivos, así como los conocimientos y saberes tradicionales, integración abordada desde la complejidad ambiental, a través del pensamiento sistémico, crítico y dialógico que propenda por la gestión ambiental y territorial.

Analizar la vulnerabilidad a procesos antropogénicos desde los enfoques tradicionales como los de riesgo-amenaza, presión-liberación y cambio global no sería lo adecuado, debido a que una evaluación bajo estos esquemas unidisciplinares o multidisciplinares limitaría la comprensión de los fenómenos y estaría ligada a la gestión del riesgo, riesgo + adaptación o a elementos políticos o económicos específicos. De igual manera, retomar elementos de tipo conceptual y metodológico trabajados comúnmente en las evaluaciones de vulnerabilidad de sistemas al cambio climático no sería lo más procedente debido a las disimilitudes dadas en las relaciones de los componentes del sistema que delimitan su sensibilidad, exposición y capacidad adaptativa; evaluaciones que trascienden principalmente hacia la gestión del riesgo y el análisis de los global a lo local (de arriba hacia abajo) para la priorización de zonas para inversión y la definición de políticas públicas asociadas a mitigación y adaptación al cambio climático.

Dichos enfoques conceptuales no incluyen los procesos socio-culturales y la complejidad de un territorio; también dejan por fuera elementos preponderantes de las comunidades humanas de la alta montaña, las cuales se caracterizan por sus procesos de conservación asociados a la mitificación y sacralización de los sistemas naturales; aspectos que determinan en parte, la sensibilidad y capacidad adaptativa territorial. Enfoques que no permiten reconocer con claridad las fuentes de exposición, sensibilidad y capacidad de adaptación que se necesitan para trabajar desde escalas detalladas a generales (de abajo hacia arriba/ bottom-up), buscando generar resultados de vulnerabilidad contextual y futura más cercanos a la realidad del territorio, la ecorregión y el país.

\section{CONCLUSIONES}

Existe, entonces, una nueva propensión a partir del enfoque de los Sistemas SocioEcológicos (SSE) para el análisis de la vulnerabilidad de los humedales de la alta montaña, que deja de lado la vieja tradición de considerar al ser humano por fuera de lo denominado como ambiente, o que es un ser superior que debe tener dominio sobre lo natural y en donde las visiones de ultranza hacia el ambientalismo o el desarrollo económico (basado en la acumulación de capital) no tienen lugar. En este sentido, las relaciones sinérgicas entre el ser humano y lo biofísico son complejas y dinámicas, que requieren ser dirigidas hacia la transición de la sostenibilidad a través de una mirada interdisciplinaria, para dar respuesta a las crisis ambientales actuales. 
Éste documento apunta a la brecha conceptual que existe en el distanciamiento del ser humano como parte de los ecosistemas lagunares, y el abordaje de su vulnerabilidad desde el enfoque de la amenaza que significa el cambio climático, dejando de lado las presiones y dinámicas humanas. En éste sentido, se invita a reconocer las lagunas de páramo como Sistemas Socio-ecológicos (SES), las cuales se caracterizan por ser cambiantes, que aprenden y se adaptan de las presiones padecidas por décadas, en donde su resiliencia en gran medida les permitirá afrontar y continuar son sus funciones ecosistémicas. Resiliencia que no es precisamente lo opuesto a ser vulnerable, sino más bien es un elemento complementario que está asociado a la capacidad adaptativa de los sistemas.

Desde esta perspectiva, es posible entender el estado y la vulnerabilidad de las lagunas paramunas ante los procesos de intervención, deterioro y conservación; al dejar de ver los cuerpos de agua como espacios intocables o por el contrario modificables, para mirarlos como un sistema lagunar dinámico que incorpora además al ser humano en su naturaleza. Finalmente, la vulnerabilidad debe analizarse como una condición sistémica, multifactorial, multisectorial, multitemporal y multiescalar.

\section{AGRADECIMIENTOS}

Este documento forma parte de una tesis doctoral en Ciencias Ambientales, que analiza desde una perspectiva interdisciplinaria, la vulnerabilidad de las lagunas de páramo a los procesos antropogénicos en el Macizo Colombiano, financiada por Colciencias a través del Fondo Francisco José de Caldas. Se da especial reconocimiento al financiador, a la Vicerrectoría de Investigaciones de la Universidad del Cauca, el Cabildo Indígena de Puracé y a Parques Nacionales Naturales de Colombia, por su apoyo en el desarrollo de esta investigación.

\section{REFERENCIAS}

[1] M. Valencia, "Vulnerabilidad de las lagunas de páramo a procesos degradativos de origen antrópico incorporando la complejidad ambiental del territorio" (Departamento del Cauca, Colombia) [Tesis Doctorado]. [Popayán (Colombia)]: Doctorado Interintitucional en Ciencias Ambientales, Universidad del Cauca, p. 303, 2014.

[2] R. Costanza, et al., "The value of the world's ecosystem services and natural capital", Nature, vol. 387, pp. 253-260, 1997.

[3] W. Mitsch and J. Gosselink, Wetlands, 3 ed. Canada: John Wiley \& Sons, Inc., 2000.

[4] R. De Groot, et al., "Valuing wetlands: guidance for valuing the benefits derived from wetland ecosystem services", Ramsar technical report, 2006.

Revista Ingenierías Universidad de Medellín, vol. 14, No. 26 pp. 29-42 ISSN 1692-3324 - enero-junio de 2015/208 p. Medellín, Colombia 
[5] IAvH, et al., Informe del estado del medio ambiente y de los recursos naturales renovables 2010. Bogotá D. C.,Colombia: Instituto de Hidrología, Meteorología y Estudios Ambientales -IDEAM-, 2011.

[6] IDEAM, Segunda Comunicación Nacional ante la Convención Marco de las Naciones Unidas sobre Cambio Climático. Bogotá, D.C., Colombia: Instituto de Hidrología, Meteorología y Estudios Ambientales -Ideam-, 2010.

[7] M. L. Parry, Climate Change 2007: Impacts, Adaptation and Vulnerability: Working Group I Contribution to the Fourth Assessment Report of the IPCC vol. 4: Cambridge University Press, 2007.

[8] M. Bedoya, et al., "Alteraciones del régimen hidrológico y de la oferta hídrica por variabilidad y cambio climático", in Estudio nacional del agua 2010, IDEAM, Ed., ed Bogotá D. C., 2010, pp. 282-320.

[9] S. R. Carpenter and K. L. Cottingham, "Resilience and Restoration of Lakes", Conservation Ecology, vol. 1, p. 2, 1998.

[10] M. Morales, et al., Atlas de páramos de Colombia. Bogotá, D. C.: Instituto de Investigación de Recursos Biológicos Alexander von Humboldt, 2007.

[11] K. C. Reiss, "Florida Wetland Condition Index for depressional forested wetlands", Ecological Indicators, vol. 6, pp. 337-352, 2006.

[12] R. Hofstede, Los páramos en el mundo: su diversidad y sus habitantes. En: Hofstede, R., Mena, P., Segarra, P. (Eds.). Los páramos del Mundo. Proyecto Atlas Mundial de los Páramos. Global Peatland Initiative/NC-IUCN/EcoCiencia. Quito, 2003.

[13] J. O. Vargas and D. Rivera, "El Páramo, un ecosistema frágil”, Cuadernos de Agroindustria y Economía rural, pp. 145-163, 1990.

[14] W. Buytaert, et al., "Human impact on the hydrology of the Andean páramos", Earth-Science Reviews, pp. 53-72, 2006.

[15] L. Naranjo, "Avifauna acuática residente y migratoria en Colombia”, in Una aproximación a los humedales en Colombia. E. Guerrero, ed, pp. 49-57, Bogotá: FEN Colombia, 1998.

[16] C. A. Vásquez, A. y Pinilla, G., "Descripción del estado trófico de diez humedales del altiplano Cundiboyacense", Universitas Scientiarum, vol. 11, pp. 61-75, 2006.

[17] E. J. Peña, et al., Evaluación de la contaminación en Ecosistemas Acuáticos: un estudio de caso en la laguna de Sonso, cuenca alta del río Cauca. Santiago de Cali: Universidad del Valle-Programa Editorial Universidad Autónoma de Occidente, 2012.

[18] M. Montenegro, "Modelling of wetland habitat availability and distribution under management alternatives : A case study of the Fúquene Lake, Colombia", Master of Science in Geoinformation Science and Earth Observation, Environmental system Analyses and Management Specialization, International Institute for Geoinformaction Science and Earth Observation (ITC), Enschede-Netherlands, 2004. 
[19] A. R. González and F. G. L. de Guevara, Plan de ordenación y manejo de la Cuenca del Lago Tota: Pontificia Universidad Javeriana, 2006.

[20] F. Muñoz, et al., "Análisis espacio temporal de humedales altoandinos: laguna de San Rafael y humedal de Calvache", in Fragmentación y coberturas vegetales en ecosistemas andinos, departamento del Cauca, A. Figueroa and M. Valencia, eds, Popayán: Universidad del Cauca, 2009.

[21] UASPNN, "Plan de Manejo Parque Nacional Natural Puracé. Informe técnico. Unidad Especial Administrativa de Parques Nacionales Naturales, Territorial SurAndina (UASPNN), ,2008.

[22] IGAC, et al., "Reporte N. 5 de áreas afectadas por inundaciones 2010-2011», 2011.

[23] IPCC, Climate Change 2001. Impacts, adaptation and vulnerability. U. K.: Cambridge University Press, 2001.

[24] B. Moya, et al., "Los humedales ante el cambio climático", Investigaciones Geográficas, pp. 127-132, 2005.

[25] Ramsar and EHAA. "Estrategia regional para la conservación y uso sostenible de humedales altoandinos", Convención de Ramsar, Gobiernos de Ecuador y Chile, CONDESAN y TNC-Chile2008.

[26] B. L. Turner, et al., "A framework for vulnerability analysis in sustainability science", Proceedings of the National Academy of Sciences of the United States of America, vol. 100, pp. 8074-8079, July 8, 2003.

[27] B. L. Turner, et al., "Illustrating the coupled human environment system for vulnerability analysis: Three case studies", Proceedings of the National Academy of Sciences of the United States of America, vol. 100, pp. 8080-8085, 2003.

[28] A. L. Luers, et al., "A method for quantifying vulnerability, applied to the agricultural system of the Yaqui Valley, Mexico", Global Environmental Change, vol. 13, pp. 255-267, 2003.

[29] B. Smit and O. Pilifosova, Adaptive Capacity and Vulnerability Reduction. Bonn, Germany, 2002.

[30] T. E. Downing, et al., "Climate Change Vulnerability: Linking Impacts and Adaptation, Report to the Governing Council of the United Nations Environmental Programme, UNEP, and Environmental Change Institute, Oxford", 2001.

[31] J. Mitchell, et al., “A contextual model of natural hazards”, Geographical Review 79, vol. 79, pp. 391-409, 1989.

[32] R. L. Ciurean, et al., "Conceptual Frameworks of Vulnerability Assessments for Natural Disasters Reduction", in Approaches to Disaster Management - Examining the Implications of Hazards, Emergencies and Disasters, J. Tiefenbacher, ed, InTech 2013.

[33] W. Adger, "Vulnerability", Global Environmental Change, vol. 16, pp. 268-281, 2006. 
[34] M. B. Soares, et al., "Conceptual elements of climate change vulnerability assessments: a review", International Journal of Climate Change Strategies and Management, vol. 4, pp. 6-35, 2012.

[35] J. Birkmann, "Risk and vulnerability indicators at different scales: Applicability, usefulness and policy implications", Environmental Hazards, vol. 7, pp. 20-31, 2007.

[36] P. C. Blaikie, et al., At risk: natural hazards, people's vulnerability, and disasters Disaster and society-from hazard assessment to risk reduction. Berlin, 1994.

[37] H. G. Bohle, et al., "Climate change and social vulnerability: Toward a sociology and geography of food insecurity”, Global Environmental Change, vol. 4, pp. 37-48, 1994.

[38] S. L. Cutter, "Vulnerability to environmental hazards", Progress in Human Geography, vol. 20, pp. 529-539, 1996.

[39] R. Chambers and G. Conway, Sustainable rural livelihoods: practical concepts for the 21st century: Institute of Development Studies (UK), 1992.

[40] L. Franco, et al., "Humedales altoandinos frente al cambio climático global. Evaluación de la vulnerabilidad y estrategia de adaptación en un complejo de humedales de la cordillera oriental colombiana: lagunas de Fúquene, Cucunubá y Palacio", Informe Final CONVENIO DHS N. ${ }^{\circ} 131$ DE 2009 ECOPETROL-Fundación Humedales, Bogota2011.

[41] H. Gitay, et al., "A Framework for assessing the vulnerability of wetlands to climate change", 2011.

[42] L. Franco, et al., "Factores de la vulnerabilidad de los humedales altoandinos de Colombia al cambio climático global”, Revista Colombiana de Geografía, vol. 22, pp. 2256-5442, 2013.

[43] N. Brooks, "Vulnerability, risk and adaptation: A conceptual framework", Tyndall Centre for Climate Change Research Working Paper, vol. 38, pp. 1-16, 2003.

[44] J. Hinkel, "Indicators of vulnerability and adaptive capacity: Towards a clarification of the science-policy interface", Global Environmental Change, vol. 21, pp. 198-208, 2011.

[45] E. Leff, Epistemología Ambiental, Cortez Editora, Sao Paulo, 2001.

[46] F. Berkes and C. Folke, "Linking social and ecological systems for resilience and sustainability”, Linking social and ecological systems: management practices and social mechanisms for building resilience, pp. 1-25, 1998.

[47] C. Folke, "Resilience: The emergence of a perspective for social-ecological systems analyses", Global Environmental Change, vol. 16, pp. 253-67, 2006.

[48] F. S. Chapin, et al., Principles of ecosystem stewardship: resilience-based natural resource management in a changing world: Cambridge Univ Press, 2009.

[49] F. Miller, et al., "Resilience and Vulnerability: Complementary or Conflicting Concepts?", Ecology and Society, vol. 15, p. 11, 2010.

[50] I. B. L. Turner, "Vulnerability and resilience: Coalescing or paralleling approaches for sustainability science?”, Global Environmental Change, vol. 20, pp. 570-576, 2010. 